\title{
Art Visualisation in the EMDR Psychotherapy Process
}

\author{
Jerome J. Leary \\ University of Brighton \\ School of Computing Engineering and Mathematics, Cockcroft Building, Brighton BN2 4GJ, UK \\ j.j.leary@brighton.ac.uk
}

\section{INTRODUCTION}

Eye Movement Desensitisation and Reprocessing (EMDR) is a psychotherapy developed by Francine Shapiro that emphasises disturbing memories as the cause of psychopathology (Shapiro 1989). It is used to alleviate the symptoms of post-traumatic stress disorder (PTSD). EMDR therapy consists of eight phases (Table 1) and each phase has its precise intention (Shapiro 1995).

Table 1: EMDR Therapy

\begin{tabular}{|l|l|}
\hline Phase & Aim \\
\hline$I$ & History and Treatment Planning \\
\hline II & Preparation \\
\hline$I I I$ & Assessment \\
\hline$I V$ & Desensitisation \\
\hline$V$ & Installation \\
\hline VI & Body Scan \\
\hline VII & Closure \\
\hline VIII & Reevaluation \\
\hline
\end{tabular}

Art therapy has successfully been used in conduction with EMDR (Breed 2013). Both techniques are body approaches and fit well together but there have been challenges when the therapeutic processing is slowed down too much, from a client's over-involvement and distraction by the art process. The art process then becomes an avoidant defence instead of a processing technique.

In 1878, the Russian physiologist Ivan Tarchanov discovered the fact that psychic states, sensory, affective, emotional, volitional, and even intellectual processes, bring about galvanic deflections. With the use of skin sensors and the electronic recording of bio-data, the emotional journey through the EMDR session could perhaps be used to produce some types of art automatically, without the involvement of the client.
Schechner has a body of work, exhibited internationally, that addresses the notion of 'vicarious trauma' (Schechner 2015). This notion, that bearing witness to the trauma of another can traumatise us is at the heart of much of his artwork. It is said that to move from a position of terrible life threatening trauma to power to rule over another people without provision put in place for the healing of that trauma can go some way to explaining the cycle of violence apparent in conflicts. Vicarious trauma suggests that this trauma is passed from generation to generation. For example, as the 'abused' Israeli state persecutes the Palestinians, the Palestinian 'abused' take out their trauma in violent acts against (often innocent) Israelis. So the cycle of abuse continues from one generation to another and from one people to another.

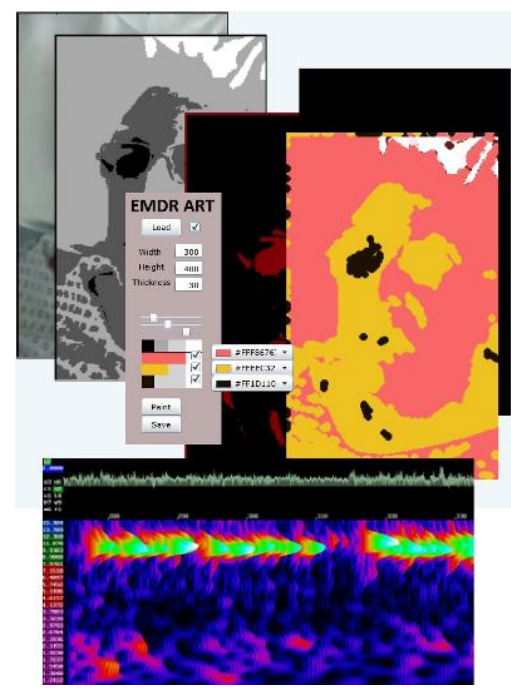

Figure 1: EMDR Art Toolkit

A collaboration between Schechner and Leary has the aim of producing art from therapy automatically. This paper describes the design of software tools for use in EMDR sessions. Video, sound and biodata would be measured and the information processed to generate graphic visualisations. The 
work produced could be used for different purposes, for example: to assist in the EMDR therapy itself by giving feedback to the therapist or bilateral stimulation of the client; or the artwork could be used for exhibition in a gallery, where the artwork attempts to visualise the trauma released during the therapy session.

\section{EMDR AND GENERATION OF ART}

\subsection{Measurements}

During the EMDR therapy three parallel recordings are made, as follows:

- Video information of the client by using a small micro-camera (e.g. 808 \#16) positioned in front of their head. Frames are captured at preset time intervals and cropped to a rectangular area around the client's eyes, and processed for horizontal eye movement. Several processing methods are used, such as grey-scale conversion, edge-detection, field of view tracking, feature correlation, contouring. The resultant paths are output in the form of coordinates into a vector array.

- Sound signals are recorded by microphone and processed for amplitude and frequency. The signals are converted into a scalar array.

- Electrocardiography and electromyography signals are collected using passive electrodes attached to the skin and monitored using an EKG-EMG device (Olimex 2015) which is interfaced to an Arduino Uno board. The signals are converted into a multidimensional scalar array (Figure 1).

\subsection{Processing Tools}

The EMDR software toolkit has been developed for:

- Sound and video recording for synchronisation with bio-signal recording

- Process digital signals in real-time using modulation and correlation. Sounds and biodata are used to amplitude-modulate the vectors from the video signal (Figure 1).

- Graphic visualisation, hue and saturation in the vector strokes, width and intensity.

- Perform range of vector transforms and processing, using contours, edge tracking and motion detection.

- Stream-control and combination of measurement signals, with user-interface functions, sliders and buttons.

- Exchange data across network using UDP. This is used for network transmission to remote machines, e.g. CNC machine.

- Control of different displays and equipment with post processors using a modified G-code language (Leary 2013).

\subsection{Output}

The toolkit enables EMDR-generated visualisations to take various forms using different painting styles. Painting options currently supported include:

- Printed painting, using inkjet or laser-print of super-imposed vector-based coloured brush strokes. This can be printed at the conclusion of the therapy session (Figure 1).

- CNC brush oil painting, using a CNC machine which paints using a brush with oil-paints in real-time on canvas (Leary 2013). The physical presence of the machine and the real-time observation of the artwork produced during the therapy session could assist in the therapeutic process.

- Light painting using a monitor or projector to simulate a photographic technique in which exposures are made using a hand-held light source while taking a long exposure photograph. This can be generated in real-time or at the conclusion of the session.

\section{CONCLUSION}

A toolkit has been developed for use in EMDR therapy to attempt to create a physical visualisation of trauma in the form of an artwork, using bio-data, video and sound measurements. This paper outlines the software processes involved and an accompanying demonstration in the exhibition shows some early examples of the work.

\section{REFERENCES}

Shapiro, F. (1989) Efficacy of the eye movement desensitisation procedure in the treatment of traumatic memories. Journal of Traumatic Stress, 2, 199-223.

Shapiro, F. (1995) Eye Movement Desensitisation and Reprocessing: Basic Principles, Protocols, and Procedures. Guilford Press, New York.

Breed, H. (2013) Integrating Art Therapy and Eye Movement Desensitization and Reprocessing to Treat Post Traumatic Stress. LMU/LLS Theses and Dissertations.

Leary, J. (2013) Development of Portable Painting Format for CNC Painting. Electronic Visualisation and the Arts (EVA 2013), London, UK, 29-31 July 2013, pp. 135-140. Electronic Workshops in Computing, British Computer Society.

Schechner, A. (2015) Postings \& Updates. http://www.dottycommies.com (retrieved 1/5/15).

Olimex (2015) Shield-EKG-EMG. Olimex. http://www.olimex.com/Products/Duino/Shields/SHI ELD-EKG-EMG/ (retrieved 1 May 15). 